\title{
Isotopic, Structural and Chemical Analyses of Pre-Solar Silicates from Asymptotic Giant Branch Stars and Type-II Supernova Explosions
}

Luc LAJAUNIE ${ }^{1}$, Manish Sanghani ${ }^{2}$, William Rickard ${ }^{3}$, Silver Sung-Yun Hsiao ${ }^{4}$, Zan Peeters ${ }^{4}$, Hsien Shang ${ }^{4}$, Der-Chuen Lee ${ }^{4}$, José Calvino ${ }^{5}$, Kuljeet Marhas ${ }^{6}$ and Martin Bizzarro ${ }^{2}$

${ }^{1}$ Departamento de Ciencia de los Materiales e IngenieríaMetalúrgica y QuímicaInorgánica, Facultad de Ciencias, Universidad de Cádiz, Cádiz, Spain, ${ }^{2}$ StarPlan - Centre for Star and Planet Formation, University of Copenhagen, Denmark, ${ }^{3}$ John de Laeter Centre, Faculty of Science and Engineering, Curtin University, Australia, ${ }^{4}$ NanoSIMS Lab, Institute of Astronomy and Astrophysics, Academia Sinica, Taiwan (Republic of China), ${ }^{5}$ Departamento de Ciencia de los Materiales e IngenieríaMetalúrgica y QuímicaInorgánica, Facultad de Ciencias, Universidad de Cádiz, United States, ${ }^{6}$ Physical Research Laboratory, India

Introduction Primitive extraterrestrial materials like carbonaceous chondrite matrices and interplanetary dust particles contain tiny dust grains that were formed in the winds of red giant branch, or asymptotic giant branch stars (AGB) and in the ejecta of novae and supernovae ( $\mathrm{SNe}$ ) explosions before the formation of our solar system. Following their formation, these tiny stardust grains of submicron size traversed through the interstellar medium before being incorporated into the cloud of gas and dust that collapsed and created our solar system. Presolar grains survived the high energy processes that created our solar system and, in their isotopic compositions, preserved the fingerprints that are the nucleosynthetic signatures of the parent stellar sources of the grains. ${ }^{1}$ Correlating isotopic data of individual presolar silicates with microstructural and chemical analyses obtained by (S)TEM, provides a unique opportunity to provide better insights into physiochemical conditions of grain formation in stellar environments, grain alteration in the interstellar and parent body processes and also helps constraining various astrophysical grain condensation models. In this work, isotopic, structural and chemical analysis of nine presolar silicate grains from the $\mathrm{CH} 3 / \mathrm{CBb} 3$ chondrite Isheyevo and CR2 chondrite NWA801 are reported. The grains studied here are found within the lithic clasts in Isheyevo and fine grained chondrule rims in NWA801 that have experienced lower amount of parent body alteration and hence the chemical compositions of presolar grains studied here are minimally altered.

Experimental Presolar oxygen anomalous grain search using oxygen isotope imaging was done in-situ using NanoSIMS 50 ion microprobe and five grains from AGB and four grains from SNe, were selected for (S)TEM investigations. The TEM lamellas were prepared using a TESCAN LYRA3 FIB-SEM at Curtin University. Structural and chemical analysis of presolar grains were performed by combining high-resolution scanning TEM imaging, spatially-resolved electron energy-loss spectroscopy (EELS) and spatially-resolved energydispersive X-ray spectroscopy (EDS) by using a FEI Titan Cubed Themis 60-300 microscope at the University of Cádiz which was operated at $200 \mathrm{kV}$. EDS quantification was corrected by using a standard reference sample of known composition and density and by taking into account the thickness of the probed area as determined

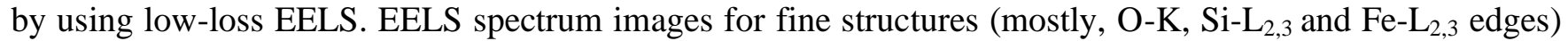
analyses were acquired with the monochromator excited allowing an energy resolution of about $0.4 \mathrm{eV}$. After denoising using principal components analysis and removal of the multiple scattering, we were able to map the heterogeneities related to the Fe oxidation states and to the oxygen local chemical environment. For the chemical mapping of the $\mathrm{Fe}^{3+} / \sum \mathrm{Fe}$ ratios, we have used a home-made Python routine based on the determination of the modified white-lines ratio. ${ }^{2}$ It allowed us to compare the degree of aqueous alteration of the grain with the surrounding rim and matrix grains.

Results TEM and STEM data have revealed a strong heterogeneity and a broad range of structural and chemical compositions of the grains that enabled us to compare the stellar grain condensation environments (e.g. AGB stars and $\mathrm{SNe}$ ), and suggest widely varying formation conditions for the presolar silicates identified in this study. Several grains are found with signatures that represent interstellar, nebular and parent body 
alteration. An oldhamite-like grain (Figure 1) within a presolar enstatite grain is probably the first observation of an oldhamite grain as a seed grain for the condensation of an enstatite grain in stellar atmospheres. This grain corresponds to a local increase in $\mathrm{Mg}$ and a local decrease in Fe with respect to the surrounding matrix. The surface of the grain surface of up to $\sim 40 \mathrm{~nm}$ is composed of higher amounts of $\mathrm{Ca}$ and $\mathrm{S}$. Below the surface of the grain and on the left side, diffusion streaks rich in Ca can be observed up to the lower boundary of the grain (on about $250 \mathrm{~nm}$ ). The diffusion of Ca could be related to thermal processes and/or aqueous alterations undergone by the grain. Figure 2 shows typical EELS chemical maps acquired on the same grain. A very thin Fe-rich rim is also seen around the enstatite grain with a $\mathrm{Fe} 3+/ \Sigma \mathrm{Fe}$ ratio of about $0.6-0.7$. The presence of several spherical nodules of Fe and Ni sulfide can also be highlighted in the matrix around the grain in the EDS and EELS chemical maps (red arrows in Figures 8 and 9). They have a diameter of about 30$45 \mathrm{~nm}$ and are similar to GEMS-like materials. Interestingly, they present a core/shell structure All these results, which will be discussed in detail, point out the importance of coordinated isotopic, microstructural and chemical studies of presolar silicates to investigate the processes that may have played a role in shaping our solar system.

Acknowledgements Authors acknowledge the use of instrumentation provided by the National Facility ELECMI ICTS ("Division de Microscopia Electronica", Universidad de Cadiz). This work has been partially financed by the Andalusian regional government (FEDER-UCA-18-106613), the European Union's Horizon 2020 research and innovation program (grant agreement 823717 - ESTEEM3) and the Spanish Ministerio de Economia y Competitividad (PID2019-107578GA-I00).

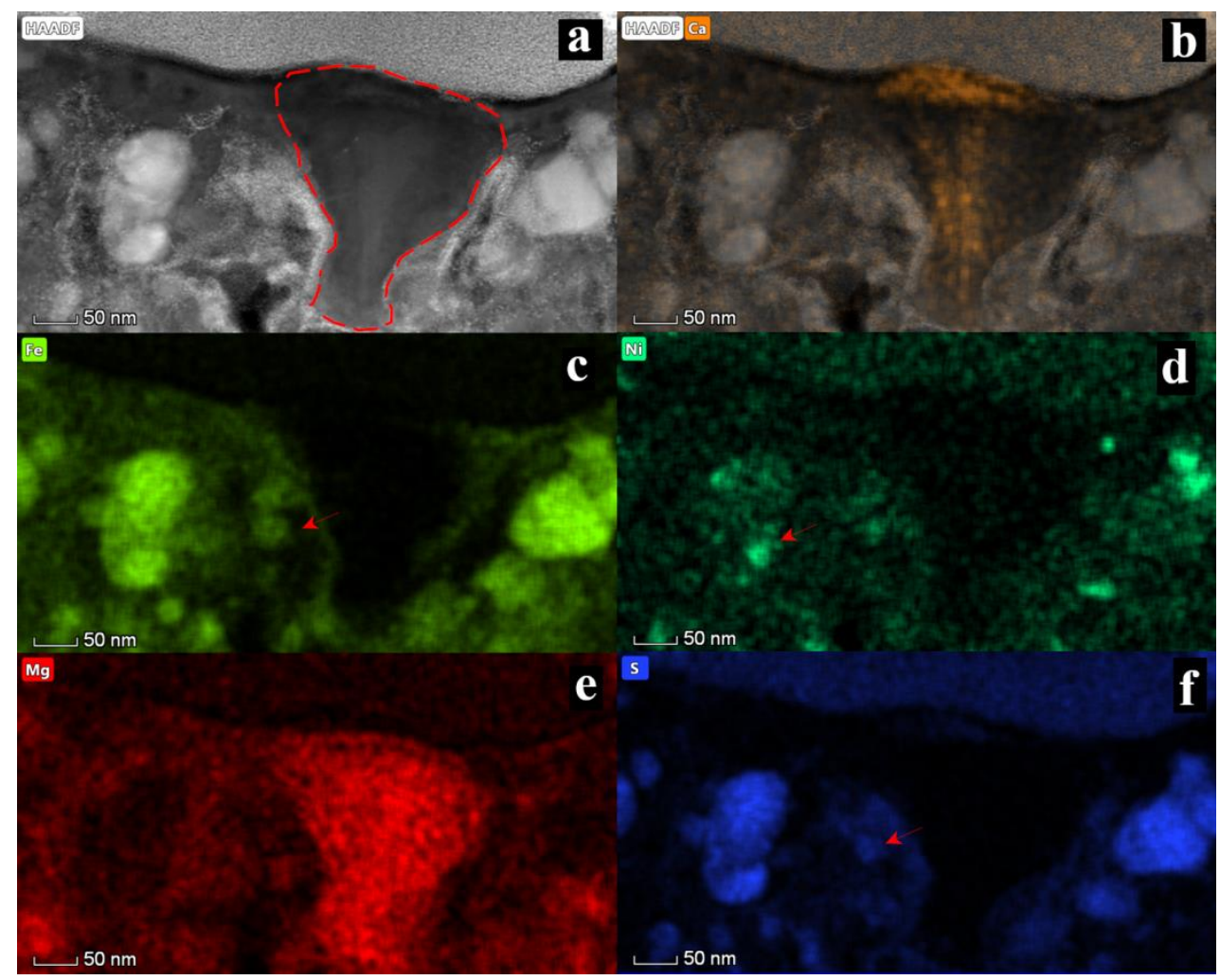

Figure 1. Figure 1. a) STEM-HAADF micrograph of the grain Isheyevo_9. The red dashed lines line highlights the boundary of the grain. b) Superposition of HAADF micrograph and Ca chemical maps derived from EDS analysis. Chemical maps derived from EDS analyses corresponding to c) $\mathrm{Fe}$, d) $\mathrm{Ni}$, e) $\mathrm{Mg}$ and f) $\mathrm{S}$ elements. The red arrows highlight the presence of GEMS-like materials. 

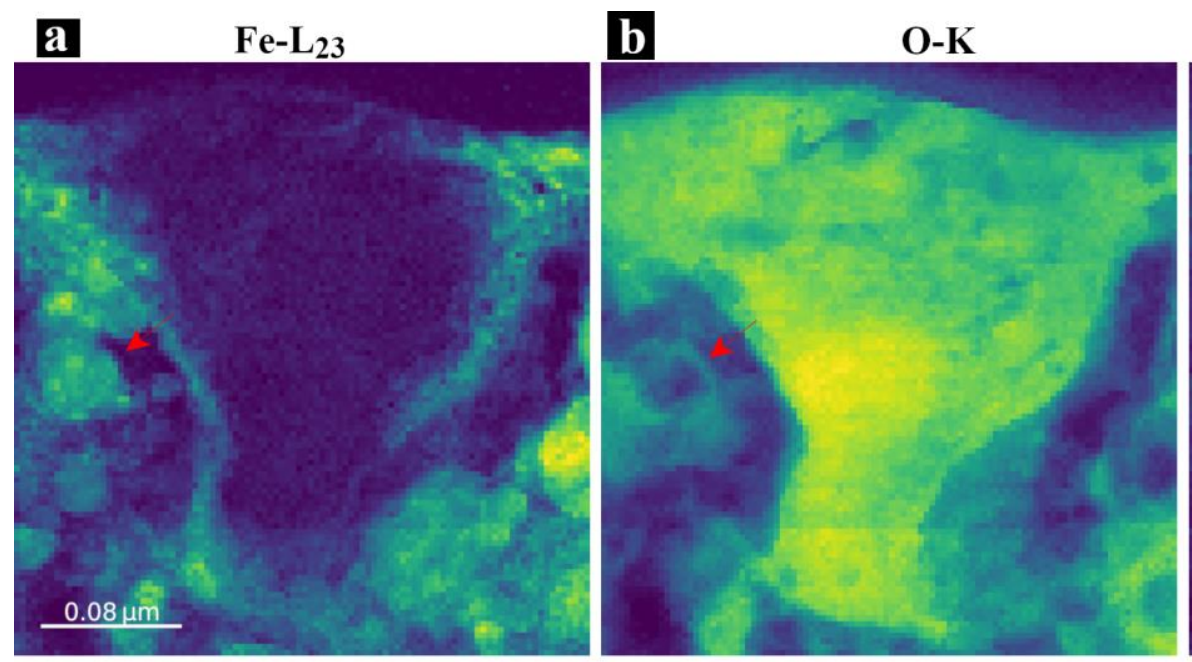

\section{c O-K pre-peak}
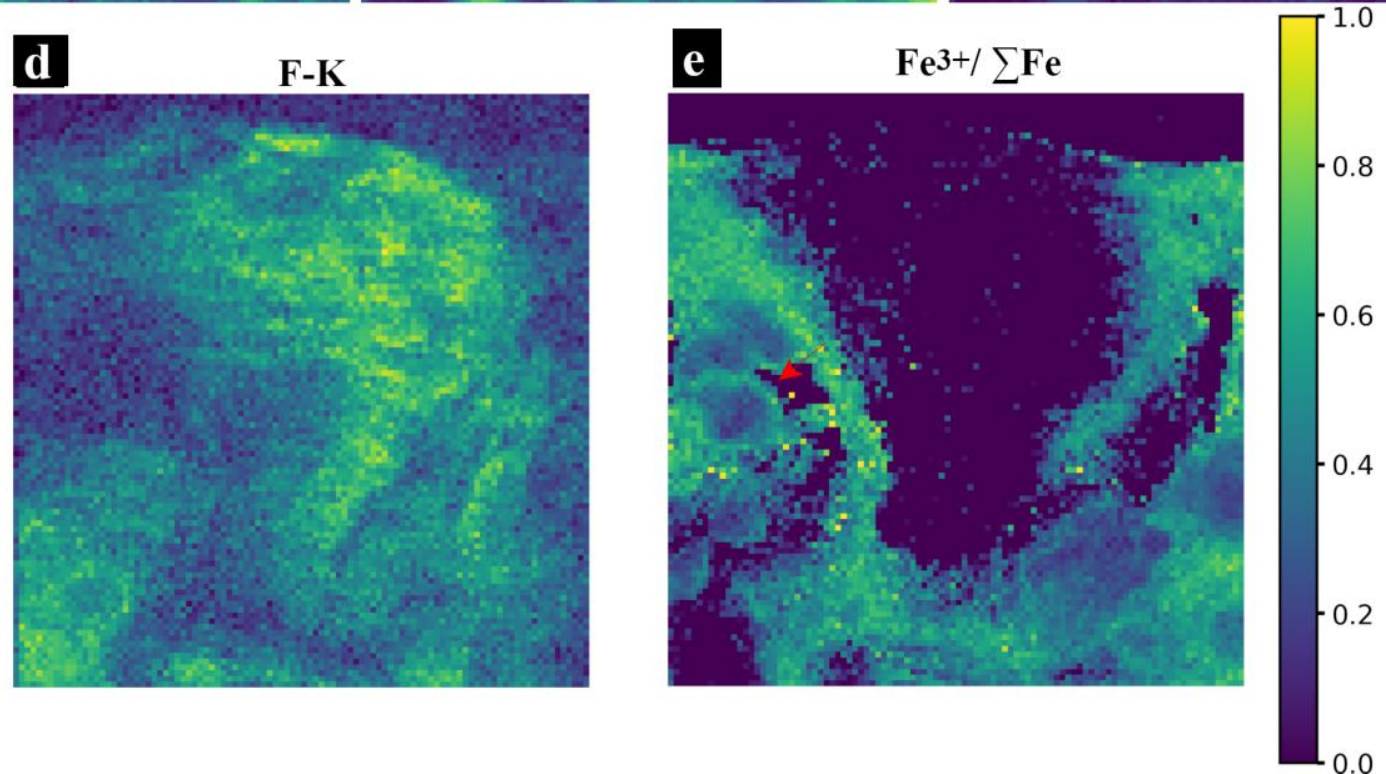

Figure 2. Figure 2. Chemical maps derived from the EELS analysis for the grain Isheyevo_9 and corresponding to a) $\mathrm{Fe}, \mathrm{b}$ ) $\mathrm{O}$, c) the $\mathrm{O}-\mathrm{K}$ pre-peak, d) $\mathrm{F}$ and e) the $\mathrm{Fe}+3 / \Sigma \mathrm{Fe}$ ratio. The red arrows highlight the presence of GEMS-like materials.

\section{References}

1. Zinner, E., (2014) Treatise on Geochemistry, Vol. 1 2nd ed. ed AM Davis

2. Van Aken, P. A., B. Liebscher, and V. J. Styrsa. "Quantitative determination of iron oxidation states in minerals using Fe L 2, 3-edge electron energy-loss near-edge structure spectroscopy." Physics and Chemistry of Minerals 25.5 (1998): 323-327. 